BULL. AUSTRAL. MATH. SOC.

VOL. 35 (1987) 153-154.

\title{
SOME ASPECTS OF THE CRITICAL PROBLEM
}

\author{
FOR MATROIDS
}

\author{
Geoffrey P. WhittLe
}

The critical problem for matroids, introduced by crapo and Rota in [2] provides a natural and unified setting for a broad range of extremal combinatorial problems. A tangential $k$-block over $G F(q)$ is a simple matroid representable over $G F(q)$ with critical exponent $k+1$ for which every loopless minor has critical exponent as most $k$. Such matroids are of central importance in the critical problem, (see for example [3] or [4]). The thesis is primarily concerned with a study of tangential $k$-blocks. The main contribution of the thesis is to develop methods by which one begins with one tangential $k$-block and constructs several others. Other topics (initially studied for their role in the critical problem) develop their own momentun. Brown truncations and Dilworth truncations of matroids receive attention as objects of interest in their own right.

In particular sufficient conditions are given for the simple matroid associated with a quotient of a tangential k-block over $G F(q)$ to be also a tangential $k$-block over $G F(q)$. The result relies heavily on the theory of modular flats of matroids developed in [1]. As a consequence it is

Received 21 April, 1986. Thesis submitted to University of Tasmania, September 1985. Degree approved February 1985. Supervisor: Dr. D.H. Row.

Copyright Clearance Centre, Inc. Serial-fee code: 0004-9727/87 $\$ A 2.00+0.00$. 
shown that there exist tangential $k$-blocks over $G F(q)$ of all ranks $r$ where $k+1 \leq r \leq q^{k}$. Characteristic polynomials of tangential $k$-blocks obtained from quotients of other tangential $k$-blocks are also studied. It is shown that if $M$ is a tangential $k$-block over $G F(q)$ and $F$ is a modular flat of $M$ which is affine over $G F(q)$ then the simple matroid associated with the complete Brown truncation of $M$ by $F$ is also a tangential $k$-block over $G F(q)$. The class of tangential $k$-blocks obtained from complete Brown truncation by modular flats of $M\left(K_{q} k_{+1}\right)$ (the matroid on the complete graph with $q^{k+1}$ vertices) is studied. It is shown that members of this class are also Dilworth truncations of certain matroids. It is shown that any tangential $k$-block over $G F(q)$ with a modular hyperplane has rank less than or equal to $q^{k}+1$ and contains $M\left(K_{k+2}\right)$ as a restriction.

\section{References}

[1] T.H. Brylawski, "Modular constructions for combinatorial geometries", Trans. Amer. Math. Soc. 203 (1975) 1-44.

[2] H.H. Crapo and G.-C Rota, On the foundations of combinatorial theory: combinatorial geometries", (M.I.T. Press, Cambridge, Mass., 1970.).

[3] D.J.A. Welsh, Colouring problems and matroids, in "Surveys in combinatorics", Lond. Math. Soc. Lecture Notes, Vol. 38

(B. Bollobas Ed. Cambridge Univ. Press, 1979).

[4] D.J.A. Welsh, "Colourings, flows and projective geometry", Niew Archief Voor Wiskunds (3), XXVIII (1980) 159-176.

\footnotetext{
Department of Mathematics, University of Tasmania, G.P.O. Box 252C, Hobart 7001

Tasmania, Aus tralia .
} 